\title{
ADAPTABILIDADE E ESTABILIDADE DE CULTIVARES DE SORGO FORRAGEIRO SEMEADOS EM DIFERENTES ÉPOCAS DO ANO ${ }^{1}$
}

\author{
ALESSANDRO GUERRA DA SILVA ${ }^{2}$, VALTERLEY SOARES ROCHA $^{3}$, COSME DAMIÃO CRUZ $^{4}$, \\ TOCIO SEDIYAMA ${ }^{5}$, GERALDO HENRIQUE FREITAS PINTO ${ }^{6}$
}

\begin{abstract}
${ }^{1}$ Extraído da Tese de Doutorado em Fitotecnia, do primeiro autor, realizada na Universidade Federal de Viçosa. Viçosa, $M G$.

${ }^{2}$ Professor da Faculdade de Agronomia, Universidade de Rio Verde. Caixa postal 104. CEP. 75901-970 Rio Verde, GO. E-mail: silvaag@fesurv.br (autor para correspondência).

${ }^{3}$ Professor do Departamento de Fitotecnia, Universidade Federal de Viçosa. CEP. 36571-000 Viçosa, MG. E-mail: vsrocha@ufv.br.

${ }^{4}$ Professor do Departamento de Biologia Geral, Universidade Federal de Viçosa. CEP. 36571-000 Viçosa, MG. E-mail: cdcruz@ufv.br.

${ }^{5}$ Professor do Departamento de Fitotecnia, Universidade Federal de Viçosa. CEP 36571-000 Viçosa, MG. E-mail: t.sediyama@ufv.br

${ }^{6}$ Engenheiro Agrônomo. CEP 35790-000. Curvelo, MG. E-mail: ricocurvelo@yahoo.com.br.
\end{abstract}

Revista Brasileira de Milho e Sorgo, v.4, n.1, p.112-125, 2005

RESUMO - Para obter informações sobre o rendimento de forragem de diferentes cultivares de sorgo forrageiro cultivados em diferentes épocas do ano efetuou-se um experimento no campo experimental do Departamento de Fitotecnia da Universidade Federal de Viçosa, durante o ano agrícola 1999/2000. No estudo foi utilizado um conjunto de oito ensaios, iniciando na primeira quinzena dos meses de outubro a maio, a partir de outubro de 1999. O delineamento experimental utilizado foi o de blocos casualizados com quatro repetições. Utilizou-se os cultivares de sorgo AG 2002, BR 501, BR 506, BR 601, BR 602 e BR 700 (forrageiros), AG 2005E e Massa 03 (duplo propósito) e AG 2501C e BRS 800 (corte e pastejo). Avaliou-se a adaptabilidade e a estabilidade do rendimento de forragem dos cultivares, utilizando o método dos trapézios quadráticos ponderados pelo coeficiente de variação. Os resultados obtidos permitiram verificar que os cultivares BR 506 e AG 2002 destacaram-se no rendimento de matéria verde, na previsibilidade de comportamento e na adaptação aos ambientes favoráveis e desfavoráveis. Para a matéria seca, o AG 2002 e o AG 2501C apresentaram maior previsibilidade de comportamento e maior adaptação aos ambientes desfavoráveis, sendo que o BR 506 e o AG 2002 foram os mais adaptados aos ambientes favoráveis. Para a proteína bruta, o AG $2501 \mathrm{C}$ e o Massa 03 destacaram-se no rendimento, na previsibilidade de comportamento e na adaptação aos ambientes desfavoráveis, sendo que o AG 2501C foi o mais adaptado aos ambientes favoráveis.

Palavras-chave: sorgo, época de semeadura, adaptação, estabilidade.

\section{ADAPTABILITY AND STABILITY OF FORAGE SORGHUM CULTIVARS SOWED IN DIFFERENT TIMES OF THE YEAR ${ }^{1}$}

\begin{abstract}
To obtain information about the forage yield of sorghum cultivars a trial was set up in the Experimental Field of the Department of Plant Science at the Universidade Federal de Viçosa, during the agricultural year of 1999/2000 to verify the yield
\end{abstract}


adaptability and stability of different forage sorghum cultivars. A group of eight experiments was used, starting in the first fortnight of October 1999 through May 2000. Each experiment was arranged in randomized blocks with four replications. The sorghum cultivars used were the AG 2002, BR 501, BR 506, BR 601, BR 602 and BR 700 (forage), AG 2005E and Massa 03 (dual purpose) and AG 2501C and BR 800 (cutting and grazing). The forage yield adaptability and stability of the cultivars were evaluated using the method of the quadratic trapezes weighted by the variation coefficient. The cultivars BR 506 and AG 2002 had a higher green matter yield, behavior predictability and adaptation to favorable and unfavorable environments. For dry matter, AG 2002 and AG 2501C presented a higher behavior predictability and adaptation to unfavorable environments, with BR 506 and AG 2002 being the most adapted to favorable environments. For crude protein, AG 2501C and Massa 03 presented a higher yield, behavior predictability and adaptation to unfavorable environments, with AG 2501C being the most adapted to favorable environments.

Key words: sorghum, sowing time, adaptation, stability.

Dentre os diversos sorgos cultivados no Brasil, existe grande variação no rendimento de forragem em resposta às diferentes épocas de semeadura. A performance diferenciada dos cultivares é atribuída às variações da temperatura e do fotoperíodo no local de cultivo. Ressalta-se ainda que, nas regiões tropicais, a baixa disponibilidade de água, associada à ocorrência de baixas temperaturas e, ou, fotoperíodos curtos são considerados limitantes ao desenvolvimento dessa gramínea durante o período de outono e inverno.

Um dos grandes problemas que se tem enfrentado na recomendação de cultivares é que, quando estes são postos a competir em várias épocas distintas, a classificação relativa entre eles pode não ser a mesma, dificultando a identificação de cultivares superiores (Ribeiro et al., 2000). Segundo Ramalho et al. (1993) para minimizar os efeitos da interação cultivares $\mathrm{x}$ ambientes, é necessário identificar cultivares mais estáveis e adaptados às condições específicas de ambiente. O cultivar que se sobressair dos demais deve apresentar, em diferentes condições ambientais, alta produtividade e sua superioridade deve ser estável. Isto auxilia, muitas das vezes, à tomada de decisão dos agricultores quanto às épocas de semeadura do sorgo, nos quais podem antecipar ou atrasar a época recomendada pelo zoneamento agroecológico da cultura.

Utilizando a metodologia de Cruz et al. (1989) e o conceito de ambientes favoráveis e desfavoráveis, alguns trabalhos têm identificado cultivares de milho (Ribeiro et al., 2000; Carvalho et al., 2001) adaptados a esses ambientes, aliado à alta previsibilidade.

As vantagens da antecipação da semeadura do sorgo têm sido demonstradas em alguns trabalhos (Avelar \& Morais, 1986; Machado et al., 1987). Porém, no Brasil, o número de informações sobre a adaptabilidade e estabilidade do sorgo é escasso. As análises de comportamento de cultivares são de extrema importância para se verificar a sua performance ao longo dos meses de cultivo.

Sendo assim, propõe-se avaliar neste trabalho a adaptabilidade e estabilidade do rendimento de forragem de cultivares de sorgo forrageiro a fim de selecionar os de melhor desempenho forrageiro para o cultivo em diferentes épocas do ano. Para isto, conduziu-se um ensaio no Campo Experimental do Departamento 
de Fitotecnia da Universidade Federal de Viçosa, em Coimbra (MG) (2051'S, 4246'W, a 720 $\mathrm{m}$ de altitude). Para auxílio na interpretação dos resultados, registrou-se os valores médios do fotoperíodo, da temperatura média e da precipitação durante o período de avaliação (Figura 1). Os fotoperíodos diários foram calculados com base na latitude e nos dias do ano (Ometto, 1981).

$\mathrm{Na}$ avaliação da adaptabilidade e estabilidade, foram utilizados os seguintes cultivares de sorgo: AG 2002, BR 501, BR 506, BR 601, BR 602 e BR 700 (forrageiros); AG 2005E e Massa 03 (duplo propósito); e AG 2501C e BRS 800 (corte e pastejo). Um conjunto de oito ensaios foi montado, sendo iniciado na primeira quinzena dos meses de outubro a maio, a partir de outubro de 1999, adotando o delineamento experimental de blocos casualizados com quatro repetições. As parcelas foram constituídas por quatro linhas, espaçadas de $0,7 \mathrm{~m}$ entre si e com 5,0 m de comprimento. Considerou-se como área útil as duas fileiras centrais, eliminado $0,5 \mathrm{~m}$ de cada extremidade, deixando-se 20 dias após a emergência, 11 plantas por metro linear para todos os cultivares (equivalente a 157.143 plantas/ha).

As adubações seguiram as recomendações feitas pela Comissão de Fertilidade do Solo do Estado de Minas Gerais (1999), sendo necessária a aplicação equivalente de $1,93 \mathrm{t} \mathrm{ha}^{-1} \mathrm{de}$ calcário dolomítico (PRNT de 76\%) três meses antes da primeira semeadura. Foi utilizado o equivalente a $500 \mathrm{~kg} \mathrm{ha}^{-1}$ da formulação 04-14-08 na semeadura e aos 30 e 45 dias após a emergência das plântulas, foi realizada a aplicação de $60 \mathrm{~kg}$ $\mathrm{ha}^{-1}$ de nitrogênio na forma de sulfato de amônio.

O corte das plantas foi realizado, rente ao solo, quando os grãos atingiram o estádio de grãos farináceos, avaliando os rendimentos de matéria verde (pesagem das plantas na área útil), matéria seca (determinação em estufa de circulação forçada de ar à $65^{\circ} \mathrm{C}$ até atingir peso constante) e proteína bruta (utilização do método de Kjeldahl para determinação do nitrogênio total). Foi realizada a irrigação de todo o ensaio, quando necessário, para que os tratamentos não fossem afetados por déficits hídricos.

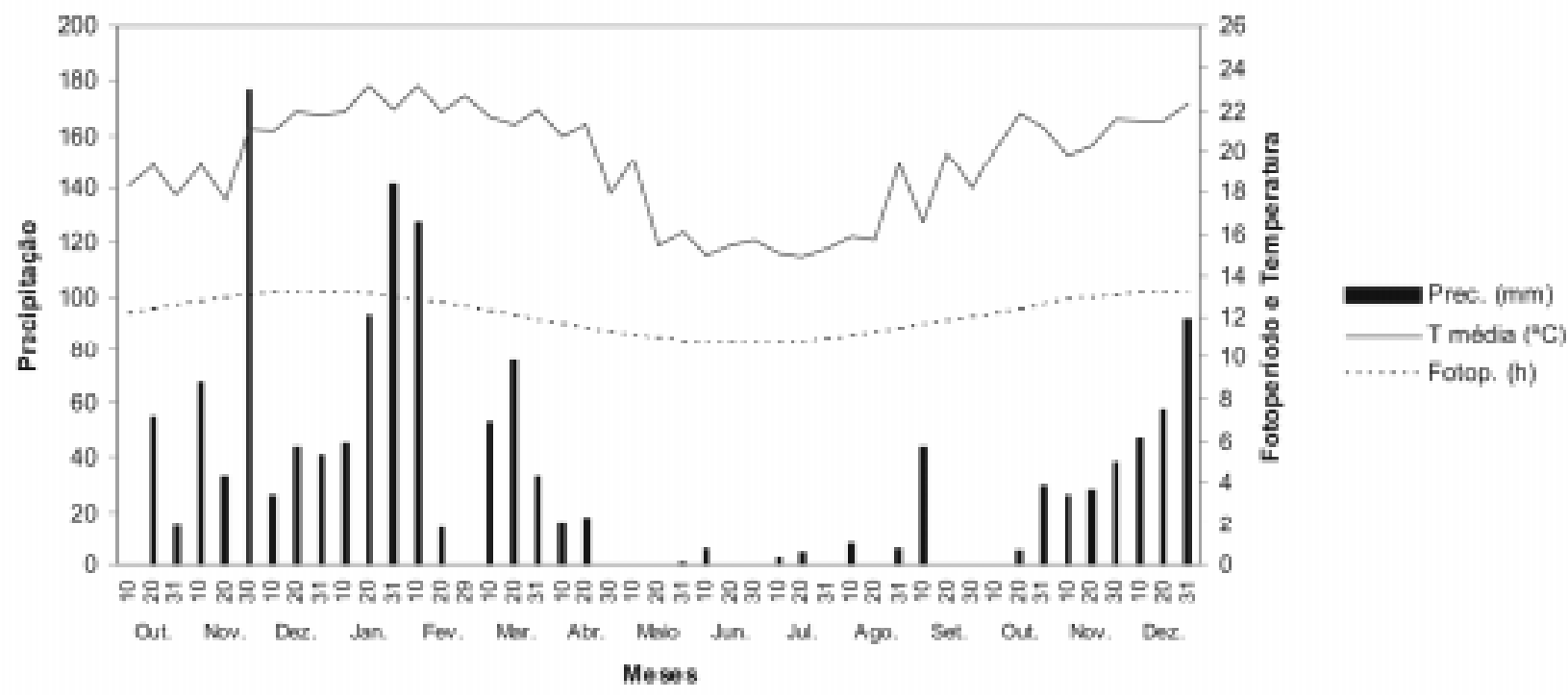

FIGURA 1. Variação do fotoperíodo, da temperatura média do ar e da precipitação pluvial, por decêndio, de outubro de 1999 a dezembro de 2000, Coimbra (MG). 
Inicialmente, efetuou-se a análise de variância para cada época de semeadura. Posteriormente realizou-se a análise conjunta para as oito épocas de semeadura, observando a homogeneidade dos quadrados médios residuais, proposto por Gomes (1990). Todas as fontes de variação, exceto o erro, foram consideradas de efeito fixo.

O método de análise de estabilidade e adaptabilidade de Lin \& Binns (1988) foi adotado, utilizando-se a estatística $\mathrm{P}_{\mathrm{i}}$, denominada de MAEC (Medida de Adaptabilidade e Estabilidade de Comportamento). Para que a recomendação de cultivares de sorgo atenda ao conceito de grupos de ambientes favoráveis e desfavoráveis, que refletem ambientes onde há condições adequadas e inadequadas ao desenvolvimento das plantas de sorgo, respectivamente, faz-se necessária a decomposição do estimador $\left(\mathrm{P}_{\mathrm{i}}\right)$ nas partes devido a ambientes favoráveis e desfavoráveis. Neste estudo, foi empregada a metodologia dos trapézios quadráticos ponderados pelo coeficiente de variação, proposta por Carneiro (1998), sendo que os meses com maior precisão experimental tiveram maior peso na estimativa da estatística MAEC. Assim, a classificação dos cultivares de sorgo foi feita com base nas estimativas dos valores de $\mathrm{P}_{\mathrm{i}}$ 's. As análises estatísticas foram realizadas empregando-se os recursos computacionais do Programa Genes (Cruz, 2001).

Quando se observa o rendimento de matéria verde dos cultivares de sorgo nas oito épocas de semeadura, verifica-se comportamento diferenciado dos materiais testados em função da resposta ao fotoperíodo e à temperatura, proporcionando variação no rendimento de forragem. Sendo o sorgo uma cultura que apresenta sensibilidade ao fotoperíodo (Karande et al., 1996; Alagarswamy et al., 1998; Craufurd \& Qi, 2001), fotoperíodos indutivos ao florescimento proporcionaram a antecipação da diferenciação floral das plantas, determinando o número de folhas produzidas que, por sua vez, proporcionou menor altura de plantas. Consequientemente, obteve-se menor rendimento de forragem. Associado a esses efeitos, a diminuição da temperatura a partir de abril (Figura 1) proporcionou a redução do desenvolvimento das plantas, ocasionando menor rendimento de matéria verde. Assim, os valores médios obtidos a partir da semeadura de janeiro foram inferiores em relação à média geral (32.804 kg ha-1 ${ }^{-1}$ Tabela 3).

O sorgo forrageiro BR 506 foi o de melhor performance para os ambientes favoráveis e desfavoráveis (menores valores de $\mathrm{P}_{\mathrm{i}}$ 's), além de apresentar o maior rendimento médio de matéria verde (49.333 $\mathrm{kg} \mathrm{ha}^{-1}$, Tabela 1). Resultados semelhantes foram obtidos com o AG 2002 e o BR 602, sendo classificados como o segundo e o quarto entre os cultivares avaliados (Tabela 2). O cultivar de duplo propósito AG 2005E apresentou o menor rendimento médio $\left(24.062 \mathrm{~kg} \mathrm{ha}^{-1}\right.$, Tabela 1), sendo classificado como o de pior performance em todos os ambientes (Tabela 2). Observou-se também a relação entre a classificação dos cultivares de sorgo com base na média do rendimento de matéria verde e os valores de $P_{i}$ geral. Os sorgos forrageiros BR 506, AG 2002, BR 602 e BR 501, acrescidos dos cultivares de corte e pastejo (AG 2501C e BRS 800) e o de duplo propósito AG $2005 \mathrm{E}$ apresentaram a mesma classificação dessas duas características (Tabela 2). Neste caso, a escolha com base no $\mathrm{P}_{\mathrm{i}}$ geral é suficiente para a recomendação destes cultivares para o rendimento de matéria verde.

No entanto, constatou-se que o posicionamento relativo, levando em consideração a média do rendimento de matéria verde, não é eficiente para a escolha dos sorgos que apresentam adaptações específicas a determinados 
ambientes. Isto pode ser comprovado com o cul- sexta para ambientes favoráveis e a nona para os tivar BR 601. Este ocupou a oitava posição no desfavoráveis (Tabela 2). Neste caso, a escolha rendimento médio, a sétima em termos gerais, a do cultivar é realizada com base em sua TABELA 1. Estimativas das médias de rendimento de matéria verde $\left(\mathrm{kg} \mathrm{ha}^{-1}\right), \mathrm{P}_{\mathrm{i}}$ geral, $\mathrm{P}_{\mathrm{i}}$ favorável e $\mathrm{P}_{\mathrm{i}}$ desfavorável, pelo método do trapézio quadrático ponderado pelo coeficiente de variação, para os cultivares de sorgo forrageiro.

\begin{tabular}{ccccc}
\hline Cultivares & Média $\left(\mathbf{k g ~ h a}^{-1}\right)$ & $\mathbf{P}_{\mathbf{i}}$ geral & $\mathbf{P}_{\mathbf{i}}$ favorável & P $_{\mathbf{i}}$ desfavorável \\
\hline BR 506 & 49.333 & $12.315,98$ & $3.750,58$ & $8.565,40$ \\
AG 2002 & 40.619 & $16.726,27$ & $6.098,31$ & $10.627,96$ \\
AG 2501C & 37.812 & $21.183,12$ & $8.668,75$ & $12.514,37$ \\
BR 602 & 33.789 & $23.898,87$ & $8.307,68$ & $15.591,19$ \\
BR 501 & 30.128 & $24.158,93$ & $7.896,59$ & $16.262,34$ \\
BRS 800 & 28.691 & $28.271,42$ & $9.725,25$ & $18.546,17$ \\
BR 601 & 28.119 & $28.959,13$ & $9.710,86$ & $19.248,26$ \\
BR 700 & 27.213 & $30.724,99$ & $13.659,36$ & $17.065,63$ \\
Massa 03 & 28.270 & $31.821,15$ & $14.247,96$ & $17.573,19$ \\
AG 2005E & 24.062 & $35.223,15$ & $14.948,48$ & $20.274,67$ \\
\hline
\end{tabular}

TABELA 2. Posição relativa dos cultivares de sorgo forrageiro quanto ao rendimento de matéria verde (RMV) avaliados pelo método do trapézio quadrático ponderado pelo coeficiente de variação.

\begin{tabular}{ccccc}
\hline Posição Relativa & RMV & Pi geral & Pi favorável & Pi desfavorável \\
\hline $1^{\underline{9}}$ & BR 506 & BR 506 & BR 506 & BR 506 \\
$2^{\underline{a}}$ & AG 2002 & AG 2002 & AG 2002 & AG 2002 \\
$3^{\underline{9}}$ & AG 2501C & AG 2501C & BR 501 & AG 2501C \\
$4^{\underline{a}}$ & BR 602 & BR 602 & BR 602 & BR 602 \\
$5^{\underline{9}}$ & BR 501 & BR 501 & AG 2501C & BR 501 \\
$6^{\circ}$ & BRS 800 & BRS 800 & BR 601 & BR 700 \\
$7^{\underline{a}}$ & Massa 03 & BR 601 & BRS 800 & Massa 03 \\
$8^{\varrho}$ & BR 601 & BR 700 & BR 700 & BRS 800 \\
$9^{9}$ & BR 700 & Massa 03 & Massa 03 & BR 601 \\
$10^{\circ}$ & AG 2005E & AG 2005E & AG 2005E & AG 2005E \\
\hline
\end{tabular}




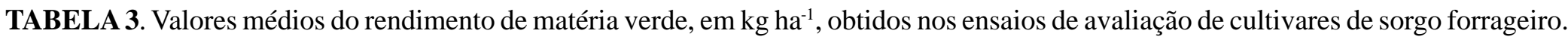

\begin{tabular}{|c|c|c|c|c|c|c|c|c|c|}
\hline \multirow{2}{*}{ Cultivares } & \multicolumn{8}{|c|}{ Ensaios } & \multirow{2}{*}{ Média } \\
\hline & Outubro & Novembro & Dezembro & Janeiro & Fevereiro & Março & Abril & Maio & \\
\hline $\mathrm{AO} 2002$ & 66.161 & 72.679 & 62.143 & 40.179 & 27.455 & 11.518 & 20.670 & 24.152 & 40.619 \\
\hline AG 2005E & 39.196 & 36.071 & 31.920 & 17.768 & 19.777 & 14.330 & 14.554 & 18.884 & 24.062 \\
\hline $\mathrm{AG} 2501 \mathrm{C}$ & 57.857 & $\$ 2.321$ & 53.304 & 31.429 & 30.312 & 19.330 & 30.804 & 27.143 & 37.812 \\
\hline BR 501 & 57.277 & 76.696 & 53.929 & 29.598 & 8.705 & 2.277 & 6.518 & 6.027 & 30.128 \\
\hline BR 506 & 87,009 & 86.473 & 60.134 & 33,036 & 27.790 & 15.670 & 43.750 & 20.804 & 49.333 \\
\hline BR 601 & 56.429 & 61.384 & 42.857 & 18.884 & 12.366 & 4.196 & 13.929 & 14.911 & 28.119 \\
\hline BR 602 & 59.062 & 73.170 & 48.750 & 27.277 & 24.598 & 6.652 & 16.116 & 14.687 & 33.789 \\
\hline $\mathrm{BR} 700$ & 38.772 & 45.937 & 38.795 & 26.429 & 23.125 & 11.384 & 15.402 & 17.857 & 27.213 \\
\hline BRS 800 & 59.710 & 46.964 & 42.857 & 19.018 & 14.687 & 9.018 & 17.589 & 19.687 & 28.691 \\
\hline Massa 03 & 40.848 & 42.187 & 32.902 & 25.491 & 25.982 & 17.366 & 18.929 & 22.455 & 28.270 \\
\hline Média & 56.237 & $59.3 \mathrm{~K}$ & 46.759 & 28.911 & 21.480 & 11.174 & 19.526 & |8.661 & 32.504 \\
\hline C.V. $(\%)$ & 7,36 & 10,80 & 7,79 & 15,48 & 9,23 & 20,85 & 18,31 & 15,64 & $=$ \\
\hline $\begin{array}{c}\text { Máximo fndice } \\
\text { Ambiental }\end{array}$ & 87.009 & 86.473 & 62.143 & 53.036 & 30.312 & 19.330 & 43.750 & 27.143 & - \\
\hline
\end{tabular}


performance no ambiente de interesse do produtor e não simplesmente na média do rendimento de todos os ambientes.

A decomposição da estatística $\mathrm{P}_{\mathrm{i}}$ geral em $\mathrm{P}_{\mathrm{i}}$ 's para ambientes favoráveis e desfavoráveis mostrou-se eficiente na discriminação dos cultivares com adaptações específicas a esses ambientes. Verificou-se para o BR 700 e o Massa 03 a elevação de duas posições para os ambientes desfavoráveis quando comparada com a recomendação geral e com a dos ambientes favoráveis (Tabela 2), conferindo maior adaptação a essas condições. Elevação de posição semelhante foi observada para o BR 501 em ambientes favoráveis em relação aos desfavoráveis e à recomendação geral, indicando maior adaptação às condições favoráveis. Por outro lado, a menor adaptação em ambientes favoráveis pode ser verificada pelos decréscimos de duas posições com o AG 2501C em relação à recomendação geral e aos ambientes desfavoráveis. Para o BRS 800, decréscimos também foram verificados para os ambientes favoráveis (uma posição) e desfavoráveis (duas posições) em relação à recomendação geral.

As posições relativas obtidas com o cultivar Massa 03 demonstram que este foi favorecido na classificação com base na média de rendimento de matéria verde em relação às estimativas de $\mathrm{P}_{\mathrm{i}}$ geral (Tabela 2). Semelhante ao observado na classificação de rendimento, esse cultivar apresentou-se melhor posicionado nos ambientes desfavoráveis quando comparado aos favoráveis e à recomendação geral. Como destacado por Ramalho et al. (1993), os cultivares podem ser adaptados a ambientes específicos e não necessariamente apresentarem altos rendimentos.

$\mathrm{Na}$ escolha do melhor sorgo para produção de matéria seca, o destaque é dado para o cultivar forrageiro AG 2002, que apresentou o terceiro maior rendimento médio e a maior previsibilidade de comportamento (Tabela 5), confirmado pelo menor valor de $\mathrm{P}_{\mathrm{i}}$ geral (Tabela 4). Esse cultivar apresentou-se como o segundo mais bem adaptado aos ambientes favoráveis e desfavoráveis (Tabela 5). O sorgo de corte e pastejo AG 2501C apresentou rendimento médio de $14.417 \mathrm{~kg} \mathrm{ha}^{-1}$ (Tabela 4) possuindo a segunda classificação para essa característica e para a recomendação geral (Tabela 5).

O sorgo AG 2501C foi o de melhor adaptação às condições desfavoráveis, sendo que nestes ambientes foi verificado maior rendimento em fevereiro e maio (Tabela 6). Nos ambientes favoráveis, devido ao menor valor de $\mathrm{P}_{\mathrm{i}}$ obtido com o BR 506 (Tabela 4), este se apresentou como o mais adaptado a estas condições.

Para o sorgo de duplo propósito AG 2005E, constatou-se a mesma posição relativa nos dois tipos de ambientes, o mesmo sendo verificado para a recomendação geral (Tabela 5). A mesma classificação obtida na recomendação geral e nos ambientes desfavoráveis foi também constatada para o BR 506, Massa 03, BR 700, BR 602 e BR 601, sendo mais adaptados nesta mesma ordem de classificação. Posições semelhantes em ambientes favoráveis e desfavoráveis também foram obtidas com o AG 2002.

Foi constatada também a relação entre a classificação do rendimento médio e o valor do $P_{i}$ geral para os cultivares AG 2501C, Massa 03 e BR 602, ocupando a segunda, a quarta e a sétima posição, respectivamente (Tabela 5). Nos ambientes favoráveis, nota-se o posicionamento diferenciado desses cultivares em relação ao rendimento médio e ao $\mathrm{P}_{\mathrm{i}}$ geral, tornando-se necessária a identificação dos cultivares de melhor adaptação a tais condições.

Além disto, observou-se também que o posicionamento relativo, levando em consideração 
TABELA 4. Estimativas das médias de rendimento de matéria seca $\left(\mathrm{kg} \mathrm{ha}^{-1}\right), \mathrm{P}_{\mathrm{i}}$ geral, $\mathrm{P}_{\mathrm{i}}$ favorável e $\mathrm{P}_{\mathrm{i}}$ desfavorável, pelo método do trapézio quadrático ponderado pelo coeficiente de variação, para os cultivares de sorgo forrageiro.

\begin{tabular}{|c|c|c|c|c|}
\hline Cultivares & Média $\left(\mathrm{kg} \mathrm{ha}^{-1}\right)$ & $P_{i}$ geral & $P_{i}$ favorávél & $P_{1}$ désfavorável \\
\hline AG 2002 & 13.426 & 730,64 & 255,97 & 474,67 \\
\hline $\mathrm{AG} 2501 \mathrm{C}$ & 14.417 & 743,97 & 316,65 & 427,32 \\
\hline BR 506 & 14.790 & 756,12 & 235,55 & 520,57 \\
\hline Massa 03 & 11.973 & 999,13 & 448,87 & 550,25 \\
\hline BR 700 & 11.213 & $1.014,91$ & 445,63 & 569,28 \\
\hline AG 2005E & 11,370 & $1.015,01$ & 413,31 & 601,70 \\
\hline BR 602 & 10.942 & $1.022,32$ & 299,11 & 723,22 \\
\hline BR 601 & 9.013 & $1.239,45$ & 433,83 & 805,62 \\
\hline BR 501 & 8.256 & $1.259,10$ & 385,69 & 873,41 \\
\hline BRS 800 & 9.243 & $1.310,64$ & 481,73 & 828,91 \\
\hline
\end{tabular}

TABELA 5. Posição relativa dos cultivares de sorgo forrageiro quanto ao rendimento de matéria seca (RMS) avaliados pelo método do trapézio quadrático ponderado pelo coeficiente de variação.

\begin{tabular}{|c|c|c|c|c|}
\hline Posição Relativa & RMS & $P_{i}$ geral & $P_{i}$ favorável & $P_{i}$ desfavorável \\
\hline $1^{\underline{\underline{Q}}}$ & BR 506 & AG 2002 & BR 506 & AG $2501 \mathrm{C}$ \\
\hline $2^{\underline{9}}$ & $\mathrm{AG} 2501 \mathrm{C}$ & $\mathrm{AG} 2501 \mathrm{C}$ & AG 2002 & AG 2002 \\
\hline $3^{\circ}$ & $\mathrm{AG} 2002$ & BR 506 & BR 602 & BR 506 \\
\hline $4^{2}$ & Massa 03 & Massa 03 & AG $2501 \mathrm{C}$ & Massa 03 \\
\hline $5^{0}$ & AG 2005E & BR 700 & BR 501 & BR 700 \\
\hline $6^{0}$ & BR 700 & AG 2005E & AG 2005E & AG 2005E \\
\hline $7^{2}$ & BR 602 & BR 602 & BR 601 & BR 602 \\
\hline $8^{2}$ & BRS 800 & BR 601 & BR 700 & BR 601 \\
\hline $9^{9}$ & BR 601 & BR 501 & Massa 03 & BRS 800 \\
\hline $10^{\circ}$ & BR 501 & BRS 800 & BRS 800 & BR 501 \\
\hline
\end{tabular}


TABELA 6. Valores médios do rendimento de matéria seca, em kg ha ${ }^{-1}$, obtidos nos ensaios de avaliação de cultivares de sorgo forrageiro.

\begin{tabular}{|c|c|c|c|c|c|c|c|c|c|}
\hline \multirow{2}{*}{ Cultivares } & \multicolumn{8}{|c|}{ Ensaios } & \multirow{2}{*}{ Médi } \\
\hline & Outubro & Novembro & Dezzembro & Janeiro & Fevereiro & Março & Abril & Maio & \\
\hline $\mathrm{AG} 2002$ & 21.223 & 22.664 & 20.480 & 14.839 & 9,879 & 4.626 & 5.811 & 7,887 & 13,426 \\
\hline $\mathrm{AG} 2005 \mathrm{E}$ & 16.616 & 16.589 & 16.423 & 8.965 & 11.120 & 6.425 & 7.452 & 7.370 & 11.370 \\
\hline AG $2501 \mathrm{C}$ & 22.603 & 18.740 & 19.317 & 13.630 & 14.082 & 6.727 & 9.289 & 10.945 & 14.417 \\
\hline BR 501 & 17.751 & 20.120 & 13.149 & 7.352 & 2.425 & 840 & 2.547 & 1.866 & 8.256 \\
\hline BR 506 & 30.947 & 23.990 & 16.779 & 13.969 & 7.755 & 5.018 & 13.442 & 6.420 & 14.790 \\
\hline BR 601 & 19.593 & 16.007 & 14.195 & 6.943 & 4.455 & 1.871 & 4.649 & 4.395 & 9.013 \\
\hline BR $60 / 2$ & 20.806 & 24.775 & 13.147 & 9.767 & 7.321 & 2.452 & 4.668 & 4.596 & 10.942 \\
\hline BR 700 & 16.762 & 15.586 & 15.100 & 12.105 & 11.460 & 4.868 & 6.395 & 7.425 & 11.213 \\
\hline BRS 800 & 20.754 & 14.899 & 11.519 & 6.183 & 5.494 & 3.566 & 5.290 & 6.242 & 9,243 \\
\hline Massa 03 & 17.505 & 15.970 & 13.940 & 12.343 & 11.858 & 7.368 & 8.552 & 8.247 & 11.973 \\
\hline Média & 20.456 & 18.934 & 15.405 & 10.610 & 8.585 & 4.376 & 6.810 & 6.539 & 11.464 \\
\hline C.V. $(\%)$ & 12,40 & 17,52 & 13,46 & 20,95 & 18,49 & 21,09 & 19,57 & 19,30 & $=$ \\
\hline $\begin{array}{l}\text { Aaxmo Indice } \\
\text { Ambiental }\end{array}$ & 30.947 & 24.775 & 20.480 & 14.839 & 14.082 & 7.368 & 13.442 & 10.945 & - \\
\hline
\end{tabular}


a média do rendimento de matéria seca, não é eficiente para a classificação dos sorgos que apresentam adaptações específicas aos ambientes. Posicionamentos diferenciados de rendimento, de $\mathrm{P}_{\mathrm{i}}$ geral e dos $\mathrm{P}_{\mathrm{i}}$ 's favorável e desfavorável foram observados para os cultivares AG 2002, BR 700, BR 601 e BRS 800 (Tabela 5). Neste caso, a escolha do cultivar é mais adequada se realizada com base na performance nos ambientes de interesse e não simplesmente na média de rendimento em todas as épocas de semeadura.

$\mathrm{Na}$ decomposição do estimador do $\mathrm{P}_{\mathrm{i}}$ geral para a variável matéria seca, verificou-se para os ambientes favoráveis a elevação do posicionamento dos cultivares BR 506, BR 602, BR 501 e BR 601 em relação à recomendação geral e aos ambientes desfavoráveis (Tabela 5). Nota-se que o BR 506 foi o mais adaptado aos ambientes favoráveis. No entanto, decréscimos em ambientes favoráveis, em relação à recomendação geral e aos ambientes desfavoráveis, foram observados para os cultivares AG 2501C, BR 700 e Massa 03 (Tabela 5). Para os ambientes desfavoráveis, a elevação de posição foi verificada para o BRS 800. Verificou-se também que este cultivar foi favorecido na classificação de rendimento em relação às estimativas de $\mathrm{P}_{\mathrm{i}}$ geral, sendo o pior classificado nesta estatística (Tabela 5). O BRS 800 apresentou-se também melhor posicionado em ambientes desfavoráveis (nona classificação) quando comparado aos favoráveis e à recomendação geral, sendo o mesmo verificado para o AG 2501C. Portanto, comprova-se mais uma vez que a escolha feita com base na média de rendimento favorece os cultivares mais produtivos em condições desfavoráveis.

$\mathrm{Na}$ avaliação da proteína bruta, a classificação dos índices ambientais elegeu as semeaduras de outubro a janeiro como ambientes favoráveis e as de fevereiro a maio, como desfavoráveis por apresentarem valores acima e abaixo da média, respectivamente (Tabela 9). Neste sentido, percebe-se que o AG 2501C destaca-se dos demais, apresentando o maior rendimento médio (1.476 kg ha-1) e a maior previsibilidade de comportamento (Tabela 7), sendo o cultivar que melhor se adaptou aos ambientes favoráveis e o segundo, nos desfavoráveis (Tabela 8). O sorgo Massa 03 apresentou o segundo maior rendimento médio, apresentando também a mesma classificação para a recomendação geral. Este cultivar foi o de melhor adaptação às condições desfavoráveis, sendo que nestes ambientes, foi verificado maior rendimento nas semeaduras de fevereiro, março e abril (Tabela 9).

Para os sorgos forrageiros BR 501 e BR 601, constataram-se a mesma posição relativa para o rendimento de proteína bruta, recomendação geral e para os ambientes favoráveis e desfavoráveis (Tabela 8). No entanto, esses cultivares se posicionaram como o décimo e o nono colocados na classificação geral, respectivamente, sendo eleitos os de pior performance.

Novamente verificou-se a elevada concordância entre a classificação dos cultivares com base na média de rendimento e na recomendação a partir da estimativa do $\mathrm{P}_{\mathrm{i}}$ geral. Além do $\mathrm{BR}$ 501 e BR 601, destacam-se os sorgos AG 2501C, Massa 03, BR 700, AG 2002, AG 2005E e BR 506 (Tabela 8). Nos ambientes favoráveis, a maior adaptação para a produção de proteína bruta foi constatada com a elevação de posição do AG 2002, BR 506 e BR 602 quando comparado com a recomendação geral e aos ambientes desfavoráveis (Tabela 8). Por outro lado, decréscimos foram verificados com os cultivares Massa 03, AG 2005E e BRS 800, confirmando a menor adaptação a esses ambientes. Em condições desfavoráveis, decréscimos em relação à recomendação 
TABELA 7. Estimativas das médias de rendimento de proteína bruta $\left(\mathrm{kg} \mathrm{ha}^{-1}\right), \mathrm{P}_{\mathrm{i}}$ geral, $\mathrm{P}_{\mathrm{i}}$ favorável e $\mathrm{P}_{\mathrm{i}}$ desfavorável, pelo método do trapézio quadrático ponderado pelo coeficiente de variação, para os cultivares de sorgo forrageiro.

\begin{tabular}{ccccc}
\hline Cultivares & Média $\left(\mathrm{kg} \mathrm{ha}^{-1}\right)$ & $\mathbf{P}_{\mathbf{i}}$ geral & $\mathbf{P}_{\mathbf{i}}$ favorável & P desfavorável $^{\text {i }}$ \\
\hline AG 2501C & 1.476 & 0,152 & 0,061 & 0,090 \\
Massa 03 & 1.386 & 0,197 & 0,111 & 0,086 \\
BR 700 & 1.349 & 0,214 & 0,100 & 0,114 \\
AG 2002 & 1.310 & 0,222 & 0,098 & 0,125 \\
AG 2005E & 1.280 & 0,250 & 0,151 & 0,099 \\
BR 506 & 1,090 & 0,322 & 0,113 & 0,208 \\
BRS 800 & 1.020 & 0,347 & 0,154 & 0,193 \\
BR 602 & 1.038 & 0,373 & 0,150 & 0,223 \\
BR 601 & 830 & 0,445 & 0,202 & 0,243 \\
BR 501 & 727 & 0,523 & 0,217 & 0,306 \\
\hline
\end{tabular}

TABELA 8. Posição relativa dos cultivares de sorgo forrageiro quanto ao rendimento de proteína bruta (RPB) avaliados pelo método do trapézio quadrático ponderado pelo coeficiente de variação.

\begin{tabular}{ccccc}
\hline Posição Relativa & RPB & Pi geral & Pi favorável & Pi desfavorável \\
\hline $1^{\underline{\underline{a}}}$ & AG 2501C & AG 2501C & AG 2501C & Massa 03 \\
$2^{\underline{a}}$ & Massa 03 & Massa 03 & AG 2002 & AG 2501C \\
$3^{\underline{a}}$ & BR 700 & BR 700 & BR 700 & AG 2005E \\
$4^{\underline{a}}$ & AG 2002 & AG 2002 & Massa 03 & BR 700 \\
$5^{\underline{a}}$ & AG 2005E & AG 2005E & BR 506 & AG 2002 \\
$6^{\underline{a}}$ & BR 506 & BR 506 & BR 602 & BRS 800 \\
$7^{\underline{a}}$ & BR 602 & BRS 800 & AG 2005E & BR 506 \\
$8^{\underline{a}}$ & BRS 800 & BR 602 & BRS 800 & BR 602 \\
$9^{\underline{a}}$ & BR 601 & BR 601 & BR 601 & BR 601 \\
$10^{0}$ & BR 501 & BR 501 & BR 501 & BR 501 \\
\hline
\end{tabular}


TABELA 9. Valores médios do rendimento de proteína bruta, em $\mathrm{kg} \mathrm{ha}^{-1}$, obtidos nos ensaios de avaliação de cultivares de sorgo forrageiro.

\begin{tabular}{|c|c|c|c|c|c|c|c|c|c|}
\hline \multirow{2}{*}{ Cultivares } & \multicolumn{8}{|c|}{ Ensaios } & \multirow{2}{*}{ Média } \\
\hline & Outubro & Novembro & Dezembro & Janciro & Fevereiro & Março & Abril & Maio & \\
\hline $\mathrm{AG} 2002$ & 1.623 & 1.911 & 1.646 & 1.796 & 1.020 & 733 & 790 & 959 & 1.310 \\
\hline AG 2005E & 1.758 & 1.584 & 1.555 & 1.045 & 1.308 & 863 & 1.136 & 986 & 1.280 \\
\hline $\mathrm{AG} 2501 \mathrm{C}$ & 2.261 & 1.919 & 1.532 & 1.683 & 1.444 & 787 & 988 & 1.194 & 1.476 \\
\hline BR 501 & 1.390 & 1.738 & 943 & 798 & 221 & 102 & 413 & 211 & 727 \\
\hline BR 506 & 1.802 & 1.749 & 1.109 & 1.447 & 476 & 467 & 1.114 & 558 & 1.000 \\
\hline BR 601 & $1.52 \mathrm{~B}$ & 1.375 & 1.005 & 819 & 445 & 258 & 697 & 513 & 830 \\
\hline BK 602 & 1.527 & 2.366 & 772 & 1.268 & 892 & 348 & 579 & 552 & 1.038 \\
\hline BR 700 & 1.716 & 2.010 & 1.420 & 1.659 & 1.326 & 662 & 1.043 & 960 & 1.349 \\
\hline BRS 800 & 2.077 & 1.592 & 1.085 & 716 & 704 & 486 & 759 & 739 & 1.020 \\
\hline Massa 03 & 1.694 & 1.581 & 1.421 & 1.587 & 1.532 & 894 & 1.325 & 1.056 & 1.386 \\
\hline Média & 1.738 & 1.783 & 1.249 & 1.282 & 937 & 560 & 885 & 773 & 1.151 \\
\hline C.V. $(\%)$ & 16,45 & 27,79 & 26,63 & 26,32 & 21,88 & 26,03 & 22,44 & 24,24 & - \\
\hline $\begin{array}{c}\text { Máximo Indice } \\
\text { Ambicntal }\end{array}$ & 2.261 & 2.366 & 1.646 & 1.796 & 1.532 & 894 & 1.325 & 1.194 & - \\
\hline
\end{tabular}


geral e aos ambientes favoráveis foram observados com os cultivares AG 2501C, BR 700, AG 2002 e BR 506, apresentando menor adaptação às condições desfavoráveis.

Os resultados de proteína bruta confirmam mais uma vez que a escolha feita com base na média de rendimento favorece os cultivares mais produtivos em condições desfavoráveis. Isto pode ser comprovado com os sorgos Massa 03, AG 2005E e BRS 800 ocupando posição superior nos ambientes desfavoráveis em relação às estimativas de rendimento médio, do $\mathrm{P}_{\mathrm{i}}$ geral e do $\mathrm{P}_{\mathrm{i}}$ para ambientes favoráveis (Tabela 8).

O uso de sorgos de maior rendimento de proteína bruta na alimentação animal proporciona maior desempenho dos animais. Neste contexto, verificou-se que os cultivares AG 2501C, Massa 03, BR 700, AG 2005E e BRS 800 apresentaram acréscimos na classificação do $\mathrm{P}_{\mathrm{i}}$ geral quando comparado aos valores de $\mathrm{P}_{\mathrm{i}}$ geral da variável matéria seca (Tabelas 8 e 5, respectivamente). Os três primeiros cultivares apresentaram também acréscimos na classificação do rendimento de proteína bruta. Estes acréscimos são atribuídos ao fato de que esses cultivares apresentam maior percentagem de proteína bruta na forragem, proporcionando maior rendimento. Em contrapartida, devido ao menor teor de proteína bruta na forragem dos sorgos BR 506 e AG 2002, foi verificado maiores decréscimos na classificação do rendimento e do $\mathrm{P}_{\mathrm{i}}$ geral.

Do exposto, conclui-se que para o rendimento de matéria verde, os sorgos BR 506 e AG 2002 foram os que se destacaram na classificação do rendimento médio, na previsibilidade de comportamento e na adaptação aos ambientes favoráveis e desfavoráveis. O AG 2002 e o AG 2501C apresentaram maior previsibilidade de comportamento para o rendimento de matéria seca e maior adaptação às condições desfavoráveis e o BR 506 e o AG 2002 foram os mais adaptados aos ambientes favoráveis. Quanto à proteína bruta, o AG 2501C e o Massa 03 se destacaram no rendimento médio, na previsibilidade de comportamento e na adaptação aos ambientes desfavoráveis e o AG 2501C foi eleito ainda como o mais adaptado às condições favoráveis.

\section{Agradecimentos}

Aos professores da Universidade Federal de Viçosa, pelas orientações e sugestões na realização deste trabalho, aos técnicos de apoio à pesquisa da referida Universidade pelo auxílio na execução das atividades e ao Conselho Nacional de Desenvolvimento Científico e Tecnológico (CNPq), pela concessão da bolsa de estudo.

\section{Literatura Citada}

ALAGARSWAMY, G.; REDDY, D. M.; SWAMINATHAN, G. Durations of the photoperiod-sensitive and insensitive phases of time to panicle initiation in sorghum. Field Crops Research, v.55, n.1-2, p.1-10, jan.1998.

AVELAR, B. C.; MORAIS, A. R. Influência das épocas de plantio na cultura do sorgo granífero em solo de cerrado. Pesquisa Agropecuária Brasileira, Brasília, v.21, n.10, p.1055-1065, out.1986.

CARNEIRO, P. C. S. Novas metodologias de análise da adaptabilidade e estabilidade de comportamento. 1998. 168f. Tese (Doutorado em Genética e Melhoramento) - Universidade Federal de Viçosa, Viçosa.

CARVALHO, H. W. L.; LEAL, M. L. S.; CARDOSO, M. J.; SANTOS, M. X.; CARVALHO, B. C. L.; TABOSA, J. N.; LIRA, M. A.; ALBUQUERQUE, M. M. Adaptabilidade e estabilidade de cultivares e híbridos de milho no 
Nordeste brasileiro no ano agrícola de 1998. Pesquisa Agropecuária Brasileira, Brasília, v.35, n.4, p.637-644, jun.2001.

COMISSÃO DE FERTILIDADE DO SOLO DO ESTADO DE MINAS GERAIS. Recomendações para o uso de corretivos e fertilizantes em Minas Gerais. 5a aproximação. Viçosa, 1999. 359 p.

CRAUFURD, P. Q.; QI, A. Photothermal adaptation of sorghum (Sorghum bicolour) in Nigeria. Agricultural and Forest Meteorology, Davis, v.108, n.3, p.199-211, 2001.

CRUZ, C. D. Programa Genes: aplicativo computacional em genética e estatística. Viçosa: UFV, 2001. 648p.

CRUZ, C. D.; TORRES, R. A.; VENCOVSKY, R. Alternative approach to the stability analysis proposed by Silva and Barreto. Revista Brasileira de Genética, Ribeirão Preto, v.12, n.13, p.567-582, 1989.

GOMES, F. P. Curso de estatística experimental. Piracicaba: NOBEL. 13.ed., 1990. 466p.

KARANDE B. I.; VARSHNEYA, M. C.; NAIDU, T. R. V. Photoperiodically sensitive time interval for panicle initiatiton of sorghum. Indian
Journal of Plant Physiology, New Delhi, v.1, n.4, p.258-261, Oct./Dec.1996.

LIN, C. S.; BINNS, M. R. A superiority measure of cultivar performance for cultivar $\mathrm{x}$ location data. Canadian Journal Plant Science, Ottawa, v.68, n.3, p.193-198, 1988.

MACHADO, J. R.; NAKAGAWA, J.; ROSOLEM, C. A.; BRINHOLI, O. Épocas de semeadura de sorgo sacarino em São Manuel e Botucatu, Estado de São Paulo. Pesquisa Agropecuária Brasileira, Brasília, v.22, n.9/10, p.951-958, set./out.1987.

OMETTO, J. C. Bioclimatologia vegetal. São Paulo: Agronômica Ceres, 1981. 440p.

RAMALHO, M. A. P.; SANTOS, J. B.; ZIMMERMANN, M. J. O.de. Interação dos genótipos $\mathrm{x}$ ambientes. In: RAMALHO, M. A. P.; SANTOS, J. B.; ZIMMERMANN, M. J. O. Genética quantitativa em plantas autógamas: aplicações ao melhoramento do feijoeiro. Goiânia: UFG, 1993. p.131-169.

RIBEIRO, P. H. E.; RAMALHO, M. A. P.; FERREIRA, D. F. Adaptabilidade e estabilidade de genótipos de milho em diferentes condições ambientais. Pesquisa Agropecuária Brasileira, Brasília, v.35, n.11, p.2213-2222, nov.2000. 